\title{
Expression levels of ARHI and Beclin1 in thyroid cancer and their relationship with clinical pathology and prognosis
}

\author{
HOUWEI ZHU* and YANQING QU* \\ Department of Thyroid Surgery, Yantaishan Hospital, Yantai, Shandong 264000, P.R. China
}

Received September 27, 2019; Accepted November 22, 2019

DOI: $10.3892 / \mathrm{ol} .2019 .11223$

\begin{abstract}
Expression levels of autophagy-related genes ARHI and Beclin1 in thyroid cancer and their relationship with clinical pathology and prognosis were investigated. The expression levels of ARHI and Beclin1 proteins in 80 cases of thyroid cancer and adjacent tissues were detected by western blot analysis. According to the expression levels of ARHI and Beclin1, low- and high-expression groups were determined and the relationship of the expression levels with the pathological parameters and prognosis in thyroid cancer was compared between the two groups. The correlation between the ARHI and Beclin1 protein expression level was analyzed by Pearson's correlation analysis. The levels of ARHI and Beclin1 proteins in thyroid cancer tissues were significantly lower than those in adjacent tissues $(\mathrm{P}<0.05)$. There was a significant difference in the expression levels of ARHI and Beclin1 in terms of pathological stage and differentiation degree of cancer tissues $(\mathrm{P}<0.001)$; however, there was no significant difference in the expression levels of ARHI and Beclin1 for different types of cancer tissues $(\mathrm{P}>0$ 05). There was a positive correlation between the expression levels of Beclin1 and ARHI ( $r=0.5187$, $\mathrm{P}<0.001)$. The 3-year survival rates of patients with low-expression level of ARHI and Beclin1 proteins were significantly lower than those of patients with high expression $(\mathrm{P}<0.05)$. In conclusion, the expression levels of Beclin1 and ARHI were low in thyroid cancer, and were significantly associated with the pathological stage, differentiation degree and prognosis in thyroid cancer. Beclin1 and ARHI can be used as predictors for the development and prognosis of thyroid cancer.
\end{abstract}

Correspondence to: Dr Houwei Zhu, Department of Thyroid Surgery, Yantaishan Hospital, 91 Jiefang Road, Yantai, Shandong 264000, P.R. China

E-mail: zdjh93@163.com

*Contributed equally

Key words: ARHI, Beclin1, thyroid cancer, clinical pathology, prognosis

\section{Introduction}

Thyroid cancer is the most common type of head and neck cancer in humans. In recent years, the incidence of thyroid cancer has risen 3-10 times, and therefore has become the focus of research on endocrine system cancer (1). The pathogenesis of thyroid cancer is not clear yet. The scope of research is mainly concentrated on diet, living environment and radiation (2). Surgery combined with chemotherapy is the common treatment regimen. Even after treatment, however, the prognosis of late undifferentiated cancer is still very poor, with an average survival time of 5 months (3). Like other cancer types, the expression levels of cancer-related genes are closely related to the occurrence and development of thyroid cancer. The expression levels of cancer-related genes regulate tumor cells to engulf their own cellular proteins or organelles into vesicles, and release lysosomes to fuse into autophagy lysosomes to decompose the inclusions, contributing to the diagnosis and treatment of thyroid cancer (4). Studies have shown that there are changes in the autophagy activity in a variety of human tumors. Through autophagy the cells of the body are more adaptable to the changes in the microenvironment, which can inhibit the differentiation of tumor cells in the early stages of tumors to a certain extent (5).

ARHI gene is one of the anti-oncogenes. According to the statistics of research data, ARHI gene is expressed in most human cells (6). ARHI is involved in tumorigenesis and the development of tumors by regulating the autophagy activity, and is mostly expressed at low levels in tumor tissues (7). Beclin1 is an anti-oncogene that participates in the formation of autophagy. Beclin1 is an important protein that regulates autophagy and is considered a marker of cell-initiated autophagy (8). A previous study has shown that autophagy and apoptosis contribute to the antitumor effect of Beclin1 in human synovial sarcoma cell line (982, SW982) (9). It has been proven that the expression levels of ARHI and Beclin1 genes are associated with the progression of thyroid cancer $(10,11)$. However, there are few studies on the relationship between ARHI and Beclin1 gene expression levels and the clinical pathology and prognosis of patients. In order to improve the understanding of thyroid cancer, the expression levels of the two genes in thyroid cancer were studied, as well as their association with clinical pathology and prognosis, to provide a basis for the development of new therapeutic methods of thyroid cancer. 


\section{Patients and methods}

General information. Eighty patients with thyroid cancer admitted to Yantaishan Hospital (Yantai, China) from January 2012 to June 2015 were selected. Thyroid cancer and adjacent tissues (within $2 \mathrm{~cm}$ from cancer tissues) were collected from all patients as experimental specimens. Among them, 35 were pathological types of papillary thyroid cancer, 20 were follicular carcinomas, 17 were undifferentiated carcinomas and 8 were medullary carcinomas. There were 44 tissues collected from patients with pathological stage I and II thyroid cancer, and 36 tissues from patients with stage III and IV cancer. The age of the study subjects ranged from 20 to 75 years. The mean age was $47.2 \pm 7.3$ years. BMI was $21.0 \pm 4.2 \mathrm{~kg} / \mathrm{m}^{2}$. There were 28 male and 52 female patients included.

\section{Inclusion and exclusion criteria}

Inclusion criteria: Patients pathologically diagnosed with thyroid cancer.

Exclusion criteria: Patients who had underwent preoperative radiotherapy and chemotherapy; patients with surgical contraindications; patients with other malignant tumor diseases; patients with severe hepatic and renal dysfunction; patients with cognitive or communication disorders; patients with poor compliance.

All patients and their families agreed to participate in the experiments and signed an informed consent form. Patients who participated in the study had complete clinical data. The study was approved by the Medical Ethics Committee of Yantaishan Hospital.

Experimental reagents and materials. The ARHI rabbit anti-human polyclonal antibody was purchased from Shanghai Huzheng Industrial Co., Ltd. (HZ-2903R). Beclin1 rabbit anti-human polyclonal antibody was purchased from Abcam (ab62557). RIPA lysate and BCA protein concentration kit were purchased from Biyuntian Technology Co., Ltd. ECL developer solution was purchased from Beijing Baier Di Diagnosis Technology Co., Ltd. Goat antihuman IgG secondary antibody labeled with horseradish peroxidase (HRP) was purchased from AmyJet Scientific, Inc. (A21050). ECL Western Blotting substrate kit was purchased from BioVision, Inc. GAPDH was purchased from ACROBiosystems (GAH-H5145). ImageJ (x64) 1.8.0 software was purchased from the National Institutes of Health.

Detection of ARHI and Beclinl protein expression levels in thyroid cancer and adjacent tissues by western blot analysis. First, the thyroid cancer and adjacent tissues were removed from the liquid nitrogen tank where they were kept. The tissues were placed on ice and cracked with RIPA lysate, and then they were placed in a water bath at $100^{\circ} \mathrm{C}$ for $10 \mathrm{~min}$ for protein denaturation. Total protein was collected and protein concentration was determined by the BCA method. The protein was separated via 10\% SDS-PAGE. Each lane was loaded with $20 \mu \mathrm{l}$ of protein and $500 \mathrm{ml}$ of electrophoresis fluid. Separated protein was subsequently transferred onto a PVDF membrane and blocked for $1 \mathrm{~h}$ at room temperature with skim milk (5\%). Next, ARHI (1:1,000), Beclin1 $(1: 1,000)$ primary antibodies

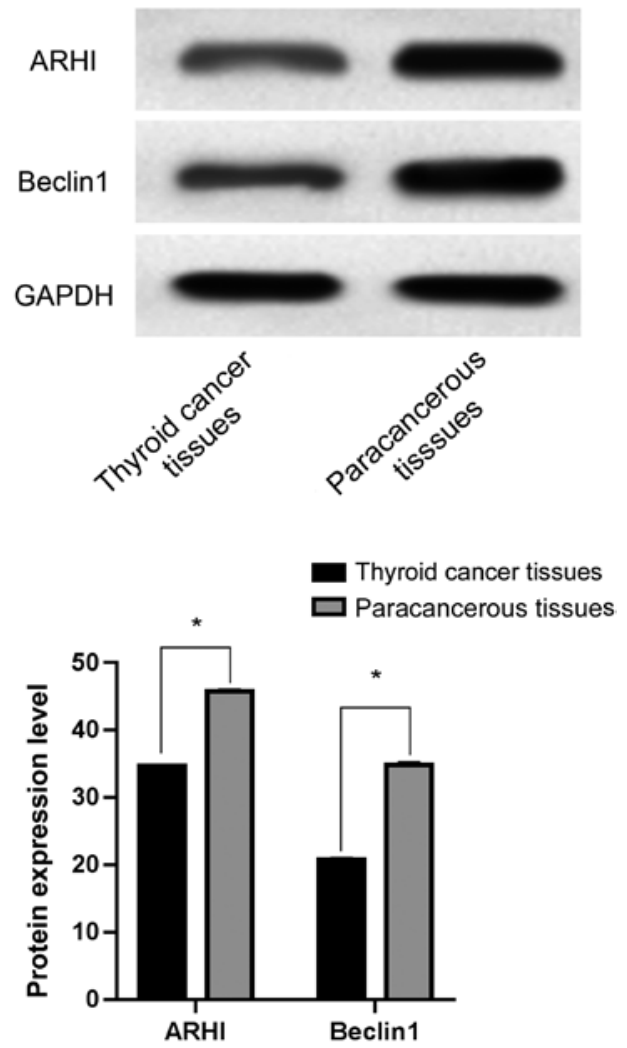

Figure 1. Expression levels of ARHI and Beclin1 in thyroid cancer and adjacent tissues. Western blot analysis revealed that the expression levels of ARHI and Beclin1 proteins in thyroid cancer were significantly lower than those in adjacent tissues. ${ }^{*} \mathrm{P}<0.05$.

and GAPDH $(1: 1,000)$ were added. The mixture was kept at $4^{\circ} \mathrm{C}$ overnight. HRP $(1: 1,000)$ was added and the membrane was incubated at $37^{\circ} \mathrm{C}$ for $2 \mathrm{~h}$. Finally, ECL developer solution was used for coloration and ImageJ software for the calculation of the grayscale or whiteness of each band, in order to semi-quantify the protein expression levels. The experiment was repeated 3 times.

Observation indices. The expression levels of ARHI and Beclin1 in thyroid cancer and adjacent tissues were compared. The relationship between the expression levels of ARHI and Beclin1 and the clinicopathological characteristics of patients with thyroid cancer was analyzed. The 3-year survival data of the patients from January 2012 to June 2015 were collected, and the relationship between the expression levels of ARHI and Beclin1 and the survival rate of the thyroid cancer patients was analyzed. The correlation between the expression levels of ARHI and Beclin1 in thyroid cancer was analyzed.

Statistical analysis. SPSS 19.0 software (IBM Corp.) was used to statistically analyze the experimental data. Chi-square test was used for the counting data. Measurement data were expressed as the mean \pm standard deviation and t-test was used for their comparison between two groups. Paired t-test was used for the comparison of the protein expression levels of ARHI and Beclin1 in tumor samples and non-cancerous adjacent tissues. One-way ANOVA followed by Bonferroni post hoc test was used for comparisons among multiple groups. Survival curves were analyzed by Kaplan-Meier survival 
Table I. Relationship between the protein expression levels of ARHI and Beclin1 and the clinicopathological characteristics of patients with thyroid cancer.

\begin{tabular}{|c|c|c|c|c|c|c|c|}
\hline Clinicopathological characteristics & $\mathrm{n}$ & ARHI & $\mathrm{t} / \mathrm{F}$-value & P-value & Beclin1 & $\mathrm{t} / \mathrm{F}$-value & P-value \\
\hline Age (years) & & & 1.826 & 0.072 & & 1.638 & 0.106 \\
\hline$\leq 55$ & 47 & $34.69 \pm 0.18$ & & & $20.76 \pm 0.25$ & & \\
\hline$>55$ & 33 & $34.61 \pm 0.21$ & & & $20.67 \pm 0.23$ & & \\
\hline $\operatorname{BMI}\left(\mathrm{kg} / \mathrm{m}^{2}\right)$ & & & 1.010 & 0.316 & & 1.486 & 0.141 \\
\hline$\leq 18$ & 31 & $34.67 \pm 0.24$ & & & $20.65 \pm 0.27$ & & \\
\hline$>18$ & 49 & $34.61 \pm 0.27$ & & & $20.74 \pm 0.26$ & & \\
\hline Pathological stage & & & 324.500 & $<0.001$ & & 303.900 & $<0.001$ \\
\hline $\mathrm{I}, \mathrm{II}$ & 44 & $45.24 \pm 0.25$ & & & $30.27 \pm 0.28$ & & \\
\hline III, V & 36 & $23.05 \pm 0.36$ & & & $12.32 \pm 0.24$ & & \\
\hline Pathological type & & & 0.302 & 0.824 & & 0.088 & 0.966 \\
\hline Papillary thyroid carcinoma & 35 & $34.64 \pm 0.16$ & & & $20.71 \pm 0.39$ & & \\
\hline Follicular carcinoma & 20 & $34.68 \pm 0.18$ & & & $20.75 \pm 0.35$ & & \\
\hline Undifferentiated carcinoma & 17 & $34.64 \pm 0.16$ & & & $20.76 \pm 0.38$ & & \\
\hline Medullary carcinoma & 8 & $34.64 \pm 0.13$ & & & $20.74 \pm 0.35$ & & \\
\hline Differentiation degree & & & 778.600 & $<0.001$ & & $5,876.000$ & $<0.001$ \\
\hline Highly differentiated & 31 & $37.49 \pm 0.25$ & & & $24.77 \pm 0.26$ & & \\
\hline Moderately differentiated & 24 & $36.39 \pm 0.38$ & & & $19.82 \pm 0.34$ & & \\
\hline Poorly differentiated & 25 & $34.14 \pm 0.33$ & & & $15.65 \pm 0.35$ & & \\
\hline Tumor diameter $(\mathrm{cm})$ & & & 1.323 & 0.190 & & 0.377 & 0.708 \\
\hline$\leq 1$ & 59 & $34.59 \pm 0.23$ & & & $20.71 \pm 0.30$ & & \\
\hline$>1$ & 21 & $34.67 \pm 0.26$ & & & $20.74 \pm 0.35$ & & \\
\hline Lymph node metastasis & & & 0.868 & 0.388 & & 10.610 & $<0.001$ \\
\hline Yes & 32 & $34.65 \pm 0.27$ & & & $20.93 \pm 0.31$ & & \\
\hline No & 48 & $34.60 \pm 0.24$ & & & $20.25 \pm 0.26$ & & \\
\hline
\end{tabular}

The values $t$ and $\mathrm{F}$ were calculated by $\mathrm{t}$-test and one-way ANOVA, respectively. BMI, body mass index.

analysis and log-rank test. Pearson's correlation test was used for correlation analysis. GraphPad Prism 8 (GraphPad Software, Inc.) was used to produce the figures. $\mathrm{P}<0.05$ was considered to indicate a statistically significant difference.

\section{Results}

Comparison of the expression levels of ARHI and BeclinI in thyroid cancer and adjacent tissues. Protein expression levels of ARHI and Beclin1 in thyroid cancer tissues were $34.62 \pm 0.25$ and $20.72 \pm 0.33$, respectively, and in adjacent tissues were $45.78 \pm 0.33$ and $34.83 \pm 0.43$, respectively. ARHI and Beclin1 protein expression levels were significantly lower in thyroid cancer tissues than those in adjacent tissues and the difference was statistically significant $(\mathrm{P}<0.05)$ (Fig. 1).

Relationship between the expression levels of ARHI and Beclinl and the clinicopathological characteristics of thyroid cancer patients. The expression of ARHI protein was associated with the pathological stage and the degree of pathological differentiation $(\mathrm{P}<0.05)$; however, there was no significant association with age, BMI, pathological classification, tumor diameter and lymph node metastasis $(\mathrm{P}>0.05)$. The expression of Beclin1 protein was associated with the pathological stage, the degree of pathological differentiation and lymph node metastasis $(\mathrm{P}<0.05)$; however, there was no significant association with age, BMI, pathological classification and tumor diameter $(\mathrm{P}>0.05)$ (Table I).

Effect of ARHI and Beclinl expression levels on the prognosis of thyroid cancer patients. According to the mean expression levels of ARHI and Beclin1 proteins, the patients were divided into an ARHI protein high-expression group (>34.62), ARHI protein low-expression group ( $\leq 34.62)$, Beclin1 protein high-expression group (>20.72) and Beclin1 protein low-expression group ( $\leq 20.72)$. There were 37 patients with high expression of ARHI protein, 43 patients with low expression of ARHI protein, 36 patients with high expression of Beclin1 protein and 44 patients with low expression of Beclin1 protein. The 3-year survival rate of patients with high and low expression of ARHI protein were 83.78 and $53.48 \%$, respectively, and the 3-year survival rate of patients with high and low expression of Beclin1 protein were 83.33 and $50.00 \%$, respectively. The 3 -year survival rates of the low-expression groups of ARHI and Beclin1 proteins were significantly lower than those of the high-expression groups $(\mathrm{P}<0.05)$ (Table II, Figs. 2 and 3). 
Table II. Relationship between the protein expression levels of ARHI and Beclin1 and the prognosis of patients with thyroid cancer.

\begin{tabular}{lcc}
\hline Groups & 3-year survival & $\chi^{2}$ value \\
\hline ARHI & & 8.320 \\
High-expression group $(\mathrm{n}=37)$ & $31(83.78 \%)$ & \\
Low-expression group $(\mathrm{n}=43)$ & $23(53.48 \%)$ & 9.004 \\
Beclin1 & $30(83.33 \%)$ & 0.002 \\
High-expression group $(\mathrm{n}=36)$ & $22(50.00 \%)$ & \\
Low-expression group $(\mathrm{n}=44)$ & & \\
\hline
\end{tabular}

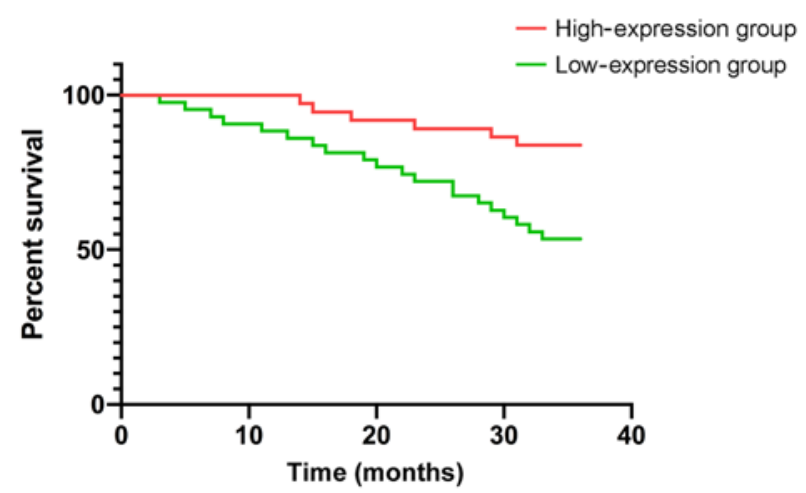

Figure 2. Relationship between ARHI expression and the prognosis of thyroid cancer patients. The 3-year survival rate of ARHI low-expression group was significantly lower than that of the high-expression group $(\mathrm{P}<0.05)$.

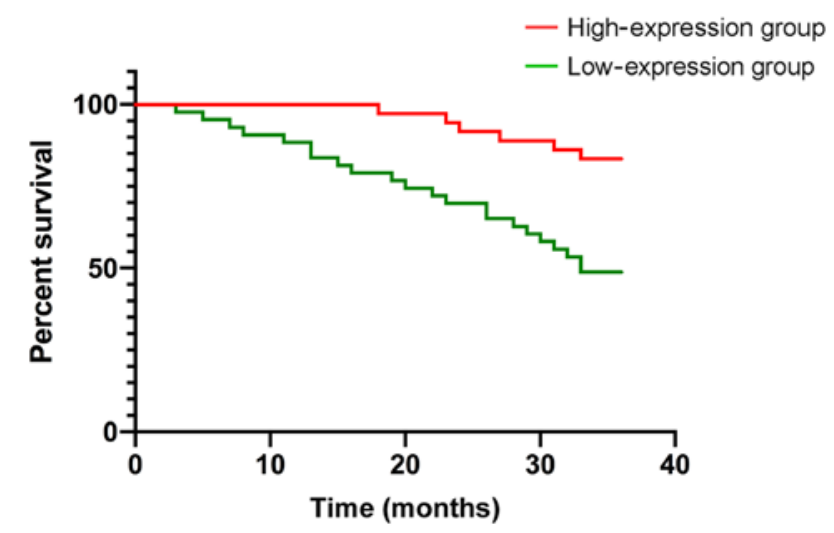

Figure 3. Relationship between Beclin1 expression and the prognosis of thyroid cancer patients. The 3-year survival rate of the Beclin1 low-expression group was significantly lower than that of the high-expression group $(\mathrm{P}<0.05)$.

Correlation analysis of the expression levels of ARHI and Beclinl in thyroid cancer. There was a positive correlation between the expression levels of Beclin1 and ARHI ( $r=0.5187$, $\mathrm{P}<0.001$ ) (Fig. 4).

\section{Discussion}

Epidemiological investigations have revealed that the incidence of thyroid tumors increases every year. However, thyroid cancer ranks first in effective treatment rate among tumor diseases, and the 5-year survival rate is still $>98 \%$. Thyroid cancer is a type of disease which is easy to be controlled

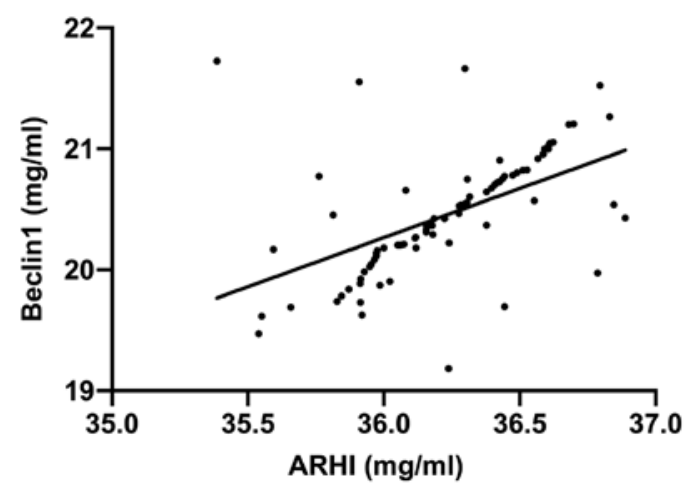

Figure 4. Correlation between the ARHI and Beclin1 expression level in thyroid cancer tissues. Pearson's correlation analysis revealed that there was a positive correlation between the protein expression levels of ARHI and Beclin1 in thyroid cancer $(r=0.5187, \mathrm{P}<0.001)$.

by routine treatment compared with other malignant tumor diseases (12); however, not all types of thyroid tumors can be treated effectively, such as tumors with distant metastasis and low differentiation, usually with poor prognosis (13). The development of thyroid cancer is mainly manifested in the imbalance of the expression levels of proto-oncogenes and anti-oncogenes. The activity of autophagy expression levels of cancer-related genes is different in different stages of the disease through autophagy. The mechanism of cancer-related genes on tumors needs to be explored in detail (14). It has been reported (15) that the genetic variation and the expression level of autophagy-related protein-5, a core participant in autophagy in thyroid cancer, is associated with the increased susceptibility of thyroid cancer and autophagy plays an important role in thyroid cancer. At present, it has been shown that ARHI can inhibit the growth of tumor cells, and the reason for inactivation of ARHI is abnormal methylation and loss of heterozygosity (16). Beclin1 can be used as a molecular regulator to repair autophagy activity (17). Previous data have shown that autophagy is related to the regulation of cell apoptosis. Autophagy is an important conservative mechanism for maintaining cell homeostasis and is closely related to the apoptosis induced by endoplasmic network (EN) stress. There is the same upstream signal transduction pathway in autophagy and apoptosis induced by EN stress; however, the relationship between them is still unclear $(18,19)$.

The results revealed that the expression levels of ARHI and Beclin1 in thyroid cancer were significantly lower than those in adjacent tissues. ARHI can inhibit the proliferation 
and activation of nuclear factor- $\kappa \mathrm{B}(\mathrm{NF}-\kappa \mathrm{B})$ in glioblastoma by decreasing the early transcription factor NF- $\kappa \mathrm{B}$ in an SmgGDS independent manner (20). The role of ARHI and its binding in mediating the activation of NF- $\mathrm{NB}$ may be a new therapy for glioblastoma. ARHI can inhibit tumors and play a role in tumor diseases, such as glioblastoma. The low expression of Beclin1 in breast, ovarian, cervical and other types of cancer has been widely reported (21). It has been shown that ARHI and Beclin1 are involved in the carcinogenesis and development of various malignant tumors. The results of the present study confirmed that ARHI and Beclin1 were involved in the occurrence and development of thyroid cancer, and both of them were expressed at low levels. The relationship between clinicopathological factors and the expression levels of ARHI and Beclin1 in thyroid cancer patients was also investigated. The results revealed that ARHI and Beclin1 were related to the different stages and grades of differentiation, whereas, there was no relationship with age, BMI and pathological type. Different types contain different subtypes, which are distinguished by differentiated and undifferentiated carcinomas. The differences in the different subtypes of the study are not obvious. Therefore, it is concluded that there is no difference between ARHI and Beclin1 among different subtypes. These results suggest that the expression levels of ARHI and Beclin1 proteins may be used in thyroid cancer, and the expression levels of autophagy proteins ARHI and Beclin1 are different for different clinicopathological characteristics of thyroid cancer patients. Next, the relationship between the expression levels of ARHI and Beclin1 and the prognosis of thyroid cancer patients was analyzed, and the results revealed that the 3 -year survival rates in the low-expression groups of ARHI and Beclin1 proteins were significantly lower than those of the high-expression groups. These results suggest that ARHI and Beclin1 proteins may be valuable in predicting the prognosis of thyroid cancer. At present, it has been found that (22) miRNA can be used as a marker for diagnosis, treatment and prognosis of thyroid cancer, and its expression is also of predictive value in the development of thyroid cancer. The evaluation of miRNA can significantly improve the accuracy of the diagnosis of thyroid cancer (23). In the present study, ARHI and Beclin1 were used to evaluate the diagnosis, treatment and prognosis of thyroid gland. In terms of clinical pathology and prognosis value, miRNA is more intuitive and accurate in the development and progression of thyroid cancer. However, whether miRNA is more suitable for the diagnosis of thyroid cancer can be determined by the detection accuracy of the expression levels of specific indicators, the ease of operation, and convenience in the later stages. Further, analysis revealed that there was a positive correlation between the expression levels of the two genes. A previous study (24) has shown that the expression levels of ARHI and Beclin1 in cervical cancer have also a positive correlation, suggesting that ARHI and Beclin1 can interact with each other through a common mechanism in a variety of malignant tumor diseases, such as thyroid cancer.

The present study focused on the expression levels of ARHI and Beclin1 in thyroid cancer and adjacent tissues, which were involved in the pathological process of thyroid cancer, as well as the prognosis of thyroid cancer. Understanding of the process of thyroid cancer can be improved by analyzing the low expression level data. However, it has not been completely elucidated how ARHI and Beclin1 regulate the microenvironment of thyroid cancer. Further research and treatment of thyroid cancer in microenvironment are required. The value of ARHI and Beclin1 in the study of the expression level of thyroid cancer is very high, mainly because the tumor cells can be killed through the function of the immune system; the microenvironment in the body of cancer patients is rich in immune cells, and immunotherapy is the ultimate treatment (25). The effects of ARHI and Beclin1 genes on the formation of thyroid tumor cells and the related mechanisms were also analyzed at the histological level of human thyroid cancer, which provided theoretical basis for the targeted treatment of thyroid cancer. Nevertheless, there are still some deficiencies in the present study. For example, there was a positive correlation between the expression levels of Beclin1 and ARHI in thyroid cancer; however, a specific explanation could not be provided and the specific interaction between these genes in thyroid cancer remains unclear. In addition, the specific impact of the two genes on thyroid cancer needs further investigation and detailed data analysis was not carried out, which constitute important aims of follow-up experiments. Also, the relatively small study group and the short follow-up period may have caused deviations in the research results to some extent. The influence of Beclin1 and ARHI on thyroid cancer and the relationship between them remain to be examined.

\section{Acknowledgements}

Not applicable.

\section{Funding}

No funding was received.

\section{Availability of data and materials}

The datasets used and/or analyzed during the present study are available from the corresponding author on reasonable request.

\section{Authors' contributions}

HZ wrote the manuscript, analyzed and interpreted the patient general data. YQ performed western blot analysis and was responsible for the analysis of the observation indicators. Both authors read and approved the final manuscript.

\section{Ethics approval and consent to participate}

The study was approved by the Medical Ethics Committee of Yantaishan Hospital (Yantai, China). Patients who participated in this research signed an informed consent form and had complete clinical data.

\section{Patient consent for publication}

Not applicable.

\section{Competing interests}

The authors declare that they have no competing interests. 


\section{References}

1. Jayarajah U, Fernando A, Prabashani S, Fernando EA and Seneviratne SA: Incidence and histological patterns of thyroid cancer in Sri Lanka 2001-2010: An analysis of national cancer registry data. BMC Cancer 18: 163, 2018.

2. Motylewska E, Stępień T, Borkowska M, Kuzdak K, Siejka A, Komorowski J, Stępień H and Ławnicka H: Alteration in the serum concentrations of FGF19, FGFR4 and $\beta$ Klotho in patients with thyroid cancer. Cytokine 105: 32-36, 2018.

3. Iyer PC, Dadu R, Gule-Monroe M, Busaidy NL, Ferrarotto R, Habra MA, Zafereo M, Williams MD, Gunn GB, Grosu H, et al: Salvage pembrolizumab added to kinase inhibitor therapy for the treatment of anaplastic thyroid carcinoma. J Immunother Cancer 6: 68, 2018.

4. Nicholson KJ and Yip L: An update on the status of molecular testing for the indeterminate thyroid nodule and risk stratification of differentiated thyroid cancer. Curr Opin Oncol 30: 8-15, 2018

5. Liu F, Gao S, Yang Y, Zhao X, Fan Y, Ma W, Yang D, Yang A and $\mathrm{Yu}$ Y: Antitumor activity of curcumin by modulation of apoptosis and autophagy in human lung cancer A549 cells through inhibiting PI3K/Akt/mTOR pathway. Oncol Rep 39: 1523-1531, 2018.

6. Fu Y, Chen J, Pang B, Li C, Zhao J and Shen K: EZH2-induced $\mathrm{H} 3 \mathrm{~K} 27 \mathrm{me} 3$ is associated with epigenetic repression of the ARHI tumor-suppressor gene in ovarian cancer. Cell Biochem Biophys 71: 105-112, 2015

7. Zou CF, Jia L, Jin H, Yao M, Zhao N, Huan J, Lu Z, Bast RC Jr, Feng Y and Yu Y: Re-expression of ARHI (DIRAS3) induces autophagy in breast cancer cells and enhances the inhibitory effect of paclitaxel. BMC Cancer 11: 22, 2011.

8. Liang C, Li W, Ge H, Zhang K, Li G and Wu J: Role of Beclin1 expression in patients with hepatocellular carcinoma: A meta-analysis. Onco Targets Ther 11: 2387-2397, 2018.

9. Xie Y, Skytting B, Nilsson G, Gasbarri A, Haslam K, Bartolazzi A, Brodin B, Mandahl N and Larsson O: SYT-SSX is critical for cyclin D1 expression in synovial sarcoma cells: A gain of function of the $\mathrm{t}(\mathrm{X} ; 18)(\mathrm{p} 11.2 ; \mathrm{q} 11.2)$ translocation. Cancer Res 62: 3861-3867, 2002.

10. Li W, Tang YX, Wan L, Cai JH and Zhang J: Effects of combining Taxol and cyclooxygenase inhibitors on the angiogenesis and apoptosis in human ovarian cancer xenografts. Oncol Lett 5: 923-928, 2013.

11. Netea-Maier RT, Klück V, Plantinga TS and Smit JW: Autophagy in thyroid cancer: Present knowledge and future perspectives. Front Endocrinol (Lausanne) 6: 22, 2015.

12. Bibbins-Domingo K, Grossman DC, Curry SJ, Barry MJ, Davidson KW, Doubeni CA, Epling JW Jr, Kemper AR, Krist AH, Kurth AE, et al; US Preventive Services Task Force: Screening for Thyroid Cancer: US preventive services task force recommendation statement. JAMA 317: 1882-1887, 2017.

13. Kim H, Kim HI, Kim SW, Jung J, Jeon MJ, Kim WG, Kim TY, Kim HK, Kang HC, Han JM, et al: Prognosis of differentiated thyroid carcinoma with initial distant metastasis: A Multicenter Study in Korea. Endocrinol Metab (Seoul) 33: 287-295, 2018.
14. Serrano A, El Haddad S, Moal F, Prazuck T, Legac E, Robin C, Brulé F, Charpentier S, Normand T, Legrand A, et al: Dysregulation of apoptosis and autophagy gene expression in peripheral blood mononuclear cells of efficiently treated HIV-infected patients. AIDS 32: 1579-1587, 2018.

15. Plantinga TS, van de Vosse E, Huijbers A, Netea MG, Joosten LA, Smit JW and Netea-Maier RT: Role of genetic variants of autophagy genes in susceptibility for non-medullary thyroid cancer and patients outcome. PLoS One 9: e94086, 2014.

16. Yakut S, Tuncer M, Berker M, Goksu E, Gurer I, Ozes O, Luleci G and Karauzum S: Aplasia ras homologous member I gene and development of glial tumors. Balkan J Med Genet 14: 37-44, 2011.

17. Kim YH, Kwak MS, Shin JM, Hayuningtyas RA, Choi JE and Shin JS: Inflachromene inhibits autophagy through modulation of Beclin 1 activity. J Cell Sci 131: 131, 2018.

18. Zhang M, Cheng YJ, Sara JD, Liu LJ, Liu LP, Zhao X and Gao H: Circulating MicroRNA-145 is associated with acute myocardial infarction and heart failure. Chin Med J (Engl) 130: 51-56, 2017.

19. Song S, Tan J, Miao Y, Li M and Zhang Q: Crosstalk of autophagy and apoptosis: Involvement of the dual role of autophagy under ER stress. J Cell Physiol 232: 2977-2984, 2017.

20. Ejaz A, Mitterberger MC, Lu Z, Mattesich M, Zwierzina ME, Hörl S, Kaiser A, Viertler HP, Rostek U, Meryk A, et al: Weight loss upregulates the small GTPase DIRAS3 in human white adipose progenitor cells, which negatively regulates adipogenesis and activates autophagy via Akt-mTOR inhibition. EBioMedicine 6: 149-161, 2016.

21. Sivridis E, Koukourakis MI, Mendrinos SE, Karpouzis A, Fiska A, Kouskoukis C and Giatromanolaki A: Beclin-1 and LC3A expression in cutaneous malignant melanomas: A biphasic survival pattern for beclin-1. Melanoma Res 21: 188-195, 2011.

22. Lodewijk L, Prins AM, Kist JW, Valk GD, Kranenburg O, Rinkes IH and Vriens MR: The value of miRNA in diagnosing thyroid cancer: A systematic review. Cancer Biomark 11: 229-238, 2012.

23. Mazeh H, Deutch T, Karas A, Bogardus KA, Mizrahi I, Gur-Wahnon D and Ben-Dov IZ: Next-generation sequencing identifies a highly accurate miRNA panel that distinguishes well-differentiated thyroid cancer from benign thyroid nodules. Cancer Epidemiol Biomarkers Prev 27: 858-863, 2018.

24. Wang ZH, Xu L, Duan ZL, Zeng LQ, Yan NH and Peng ZL: Beclin 1-mediated macroautophagy involves regulation of caspase-9 expression in cervical cancer HeLa cells. Gynecol Oncol 107: 107-113, 2007.

25. Mould RC, van Vloten JP, AuYeung AW, Karimi K and Bridle BW: Immune responses in the thyroid cancer microenvironment: Making immunotherapy a possible mission. Endocr Relat Cancer 24: T311-T329, 2017.

This work is licensed under a Creative Commons Attribution-NonCommercial-NoDerivatives 4.0 International (CC BY-NC-ND 4.0) License. 